\title{
Oncolytic herpes simplex virus treatment of metastatic breast cancer
}

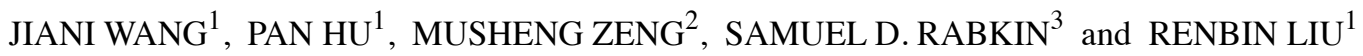 \\ ${ }^{1}$ Breast Cancer Center, The Third Affiliated Hospital; ${ }^{2}$ Department of Laboratory Medicine, Tumor Hospital, \\ Sun Yat-sen University, Guangzhou, P.R. China; ${ }^{3}$ Molecular Neurosurgery Laboratory, \\ Massachusetts General Hospital, Harvard Medical School, Boston, MA, USA
}

Received September 1, 2011; Accepted October 18, 2011

DOI: $10.3892 /$ ijo.2011.1266

\begin{abstract}
The high prevalence and poor prognosis of breast cancer provides a strong rationale for developing new treatment strategies and preventive and therapeutic agents. Oncolytic replication-competent herpes simplex virus (HSV) can infect tumor cells, replicating and killing the cells by direct cytopathic effect and then spreading within the tumor. Replication of oncolytic HSV leads to the destruction of the infected tumor cell and release of new virions, which are able to infect adjacent cells until potentially all tumor cells are destroyed. In this study, the cytotoxicity of a third-generation oncolytic HSV vector, designated $\mathrm{G} 47 \Delta$, was examined in human breast cancer cell lines, as well as in immortalized and normal breast cells. A pulmonary metastatic model of breast cancer established in $\mathrm{Balb} / \mathrm{c}$ nude mice was used to evaluate the efficacy of G47 $\Delta$ treatment. Systemic treatment by intravenous administration of G47 $\Delta$ for metastatic lung tumors was initiated 14 days after injection of tumor cells. On Day 56, the mice were sacrificed and tumor nodules on the surface of the lung were counted. G47 $\Delta$ was highly cytotoxic to breast cancer and immortalized breast cells in vitro at low multiplicities of infection (MOI), while normal breast cells remained viable 5 days after infection. In the pulmonary metastatic model, the average number of surface lung tumor nodules in the G47 $\Delta$-treated group was approximately 9 -fold less than in the control-treated group. $\mathrm{X}$-gal staining illustrated viral replication and spread in the tumor cells in vitro and in vivo. In conclusion, G47 $\Delta$ effectively killed human breast cancer cells and immortalized breast cells but not normal breast cells. Systemic administration of G47D by tail vein injection was effective in inhibiting the growth of established breast cancer lung metastases.
\end{abstract}

Correspondence to: Dr Renbin Liu, Breast Cancer Center, The Third Affiliated Hospital, Sun Yat-sen University, 600 Tianhe Road, Guangzhou 510630, P.R. China

E-mail: liur@vip.163.com

Key words: breast cancer, pulmonary metastatic breast cancer, oncolytic herpes simplex virus, immortalized breast cells, pulmonary metastatic model

\section{Introduction}

Breast cancer is a polygenetic disorder with complicated inheritance factors, leading to a complex etiology and prognosis. Breast cancer ranks among the most frequently diagnosed malignancies afflicting women worldwide (World Health Organization 2008). Metastasis, which in essence determines prognosis, is a common characteristic of malignant tumors. It is a multi-factorial, non-random and sequential process. Breast cancer metastasis to distant organs, such as lung, liver, brain and bone, is the leading cause of mortality. Unfortunately, available technologies for the early detection of breast cancer metastases are limited. Thus, although great progress has been made in improving the survival rate of patients diagnosed with breast cancer, it still has a very high mortality rate.

The high prevalence and poor prognosis of breast cancer provides a strong rationale for developing new treatment strategies and preventive and therapeutic agents. The concept of using viruses as a cancer treatment dates back to the beginning of the last century, when it was observed that patients with different kinds of malignant tumors who underwent rabies vaccination or experienced viral diseases exhibited spontaneous tumor regression (1). However, the use of wild-type viruses for cancer therapy was associated with serious adverse events and it was only with genetic-engineering of virus vectors to reduce pathogenicity that oncolytic virus therapy became a promising therapeutic strategy (2). Replication-competent oncolytic HSV-1 vectors target actively dividing neoplastic cells while sparing normal cells and can be exploited as a therapeutic strategy for the selective destruction of tumors without damaging adjacent normal tissue. The infected neoplastic cells, which are killed by the replicating virus, release progeny virions. This pattern of viral amplification and lateral cell-to-cell transmission results in the further destruction of surrounding tumor cells. As these viruses destroy tumor cells by oncolysis, cross-resistance with other therapy approaches, such as radiotherapy, chemotherapy and hormonal therapy, typically does not arise. Thus viral therapy can serve as a complement to traditional therapeutic strategies.

A variety of oncolytic HSV vectors have been developed as therapeutic antitumor agents; the vector used in this study, $\mathrm{G} 47 \Delta$, is a third generation oncolytic HSV vector. It has three main mutations in its genome $(\gamma 34.5, \mathrm{ICP} 6, \alpha 47)$ that restrict 
G47A cytotoxicity to tumor cells. $\gamma 34.5$ is the major gene determinate of HSV neurovirulence (3). The protein product of $\gamma 34.5$ precludes the shut-off of host protein synthesis in infected cells (4). ICP6 is the large subunit of viral ribonucleotide reductase, a key viral enzyme for DNA synthesis that is necessary for virus replication in normal non-dividing cells (5). These two mutations, also present in G207, restrict virus replication to tumor cells, attenuate neuropathogenicity and make spontaneous reversion nearly impossible (6). In addition, the mutation in ICP6 makes the virus more sensitive to acyclovir and ganciclovir (6), which is an added safety feature for clinical use. Oncolytic HSV is also capable of inducing an antitumor immune response (7-9), which is enhanced with G47 $\Delta$ because of the deletion of $\alpha 47$, which blocks peptide loading of MHC class I molecules (10). Thus, increasing MHC class I presentation, stimulating lymphocytes and decreasing NK cytolysis of infected cells $(11,12)$, which can enhance the antitumor immune response. Antitumor immune responses are likely to be particularly important for treating metastatic tumors. Deletion of $\alpha 47$ also complements the attenuated growth of $\gamma 34.5$-mutants by blocking the shut-off of host protein synthesis (13).

Previously, we have demonstrated that G47 $\Delta$ was efficacious for the treatment of: subcutaneous breast cancer tumors formed by the implantation of MDA-MB-435 human breast cancer cells (14); intracerebral tumors with the M6c murine breast cancer cells; and spontaneously arising mammary tumors in heterozygous $\mathrm{C} 3(1) / \mathrm{T}-\mathrm{Ag}$ transgenic mice (15). The goal of this study was to evaluate the cytotoxicity of G47 $\Delta$ in three human breast cancer cell lines, two immortalized human breast cell lines and two primary cultured normal breast cells, as well as the efficacy of systemically administered G47 $\Delta$ in a pulmonary metastatic breast cancer model. To our knowledge, this study is the first to describe the treatment of pulmonary breast cancer metastases with G47D.

\section{Materials and methods}

Cells and virus. Human breast cancer cell lines MDA-MB435, SK-BR-3, MDA-MB-453 and MCF-7 (all from State Key Laboratory of Oncology in Southern China, Sun Yat-sen University Cancer Center, Guangzhou, China) as well as Vero cells (African green monkey kidney; purchased from The Committee on Type Culture Collection of Chinese Academy of Sciences, Shanghai, China) were cultured in DMEM with glucose (4.5 g/l; Mediatech, Inc., Herndon, VA) supplemented with $10 \%$ fetal calf serum (Hyclone Laboratories, Logan, $\mathrm{UT})$ at $37^{\circ} \mathrm{C}$ in $5 \% \mathrm{CO}_{2}$. Immortalized human breast cells MCF-10A and 76N-tert (all from State Key Laboratory of Oncology in Southern China) were cultured in keratinocyte/ serum-free medium (Invitrogen, Grand Island, NY) at $37^{\circ} \mathrm{C}$ in $5 \% \mathrm{CO}_{2}$.

Oncolytic HSV G47D was grown in Vero cells in DMEM containing $3 \%$ inactivated fetal calf serum at $34.5^{\circ} \mathrm{C}$. Cells were infected at a low multiplicity of infection (MOI) of 0.02-0.03, and when total CPE (cytopathic effect, cells are rounded and refractile) occurred, infected cells were harvested. Cells were resuspended in a 1:1 mix of DMEM (no serum) and virus buffer (150 mM NaCl/20 mM Tris, $\mathrm{pH} 7.5)$ and the cell suspension was rapidly frozen in EtOH/dry ice. The suspen- sion went through 3 rounds of freeze-thawing to lyse cells for virus release and cell debris was removed by low-speed centrifugation $\left(2000 \mathrm{~g}\right.$ for $10 \mathrm{~min}$ at $4^{\circ} \mathrm{C}$ ). The titer of $\mathrm{G} 47 \Delta$ was determined on Vero cells by plaque assay.

Primary cultures of human mammary epithelia cells (HMECs). The procedure for primary culture is similar to that for primary human nasopharyngeal epithelial cells (16). Briefly, fresh normal breast tissues adjacent to benign breast disease were obtained from patients who were devoid of breast carcinoma at the Breast Cancer Center, Third Affiliated Hospital of Sun Yat-sen University, Guangzhou, China. Prior consent of the patients and approval from the Sun Yat-sen University Institute Research Ethics Committee were obtained for the use of these tissue samples. One part of the tissue was used for primary culture of epithelial cells, and the remaining tissue was fixed with formaldehyde and embedded in paraffin to ensure pathologically normal tissue. To initiate primary culture of HMECs, the breast tissues were cut into $\sim 1-\mathrm{mm}^{3}$ explants with scissors and placed into T-25 culture flasks (Falcon) containing $2 \mathrm{ml}$ of keratinocyte/ serum-free medium. The cells that grew out from the biopsies were named HMEC1 and HMEC 2 and characterized with a mouse anti-cytokeratin antibody (Zhongshan Goldenbridge Biotechnology, Beijing, AE1/AE3, ZM-0069).

In vitro cytotoxicity. Cells were seeded into 6-well plates at a density of $1 \times 10^{5}$ cells per well and after a 24 -h incubation at $37^{\circ} \mathrm{C}$ were infected with $\mathrm{G} 47 \Delta$ at a MOI of 0.01 and 0.1 . The controls were mock infected with phosphate-buffered saline (PBS). The infected cells were incubated at $37^{\circ} \mathrm{C}$ for 5 days, with the number of surviving cells counted every day with a hemocytometer. Before counting, cells were washed twice with $1 \mathrm{ml}$ of PBS to eliminate floating cells and then trypsinized. Trypan blue staining was used to exclude dead cells. The average number of cells (from duplicate wells) was plotted as a percent of the mock wells. X-gal staining was performed every day to identify infected cells.

$X$-gal histochemistry. G47D expresses $\beta$-galactosidase, thus infected cells are stained blue by X-gal staining. Culture medium was removed and cells were fixed with $0.2 \%$ glutaraldehyde/2\% paraformaldehyde for $5 \mathrm{~min}$. After 3 washes with PBS, cells were incubated with X-gal substrate solution [PBS (pH 7.2), containing $0.5 \mathrm{mg} / \mathrm{ml}$ 5-bromo-4-chloro-3-indolyl$\beta$-D-galactopyranoside, $5 \mathrm{mmol} / 1$ potassium ferricyanide, $5 \mathrm{mmol} / \mathrm{l}$ potassium ferrocyanide and $2 \mathrm{mmol} / 1$ magnesium chloride] at $37^{\circ} \mathrm{C}$ for $2 \mathrm{~h}$.

Animal studies. Four-week-old female Balb/c nude mice were purchased from Shanghai Institutes for Biological Sciences, CAS, Shanghai, China. All animal procedures followed the Institutional Guidelines of Guangdong Province and were approved by the Use Committee for Animal Care and the Sun Yat-sen University Institute Research Ethics Committee.

Metastatic lung tumors were established by tail vein injection of MDA-MB-435 cells $\left(1 \times 10^{6} / 100 \mu \mathrm{l}\right)$ and treated by tail vein injection of G47 $\left(2 \times 10^{7} \mathrm{pfu} / 100 \mu \mathrm{l}\right)$ or virus buffer (PBS) on Days 14, 17, 21 and 24 post-tumor implantation. Mice were monitored for signs of morbidity and weighed twice a week. 

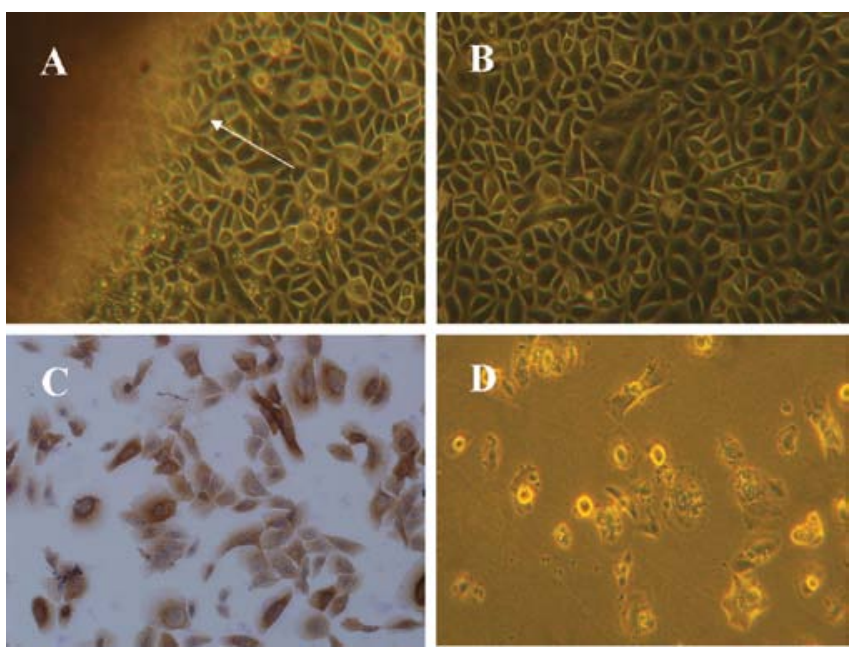

Figure 1. Establishment of HMEC culture. (A) Primary culture of HMEC1 (the arrow shows edge of biopsy). (B) Morphology of HMEC1 (passage 2). (C) Positive staining of cytokeratin in HMEC1 cytoplasm with the nucleus stained by hematoxylin. (D) Morphology of cells undergoing senescence and death.

Treated mice were sacrificed on Day 56, and the lungs were excised in their entirety and weighed. Lungs were then stained with India ink through the trachea and metastatic lesions (tumor nodules - white spots against the black background) counted. The lungs were photographed, fixed in formaldehyde, embedded in paraffin and processed for histology after hematoxylin-eosin staining.
Viral biodistribution in lung metastases. A second group of 10 mice was used for virus biosdistribution in the lung metastases. Tumors were established as above and treated with G47 $\Delta$ $\left(2 \times 10^{7} \mathrm{pfu} / 100 \mu \mathrm{l}\right)$ on Days 44 and 47 post-tumor implantation. Two mice were sacrificed on each of the following Days: 48 , $50,53,56$ and 59. Next, lungs were removed, embedded in OCT, immediately frozen on dry ice and $10-\mu \mathrm{m}$ thick cryostat sections were cut. The sections were fixed with $2 \%$ paraformaldehyde in PBS for $10 \mathrm{~min}$, followed by incubation with PBS containing $2 \mathrm{mmol} / \mathrm{l}$ magnesium chloride, $0.01 \%$ sodium deoxycholate and $0.02 \%$ NP40 for $10 \mathrm{~min}$ at $4^{\circ} \mathrm{C}$. Finally, sections were stained with X-gal substrate solution [PBS (pH 7.2), containing $1 \mathrm{mg} / \mathrm{ml}$ 5-bromo-4-chloro-3-indolyl-h-Dgalactopyranoside, $5 \mathrm{mmol} / \mathrm{l}$ potassium ferricyanide, $5 \mathrm{mmol} / 1$ potassium ferrocyanide, $2 \mathrm{mmol} / \mathrm{l}$ magnesium chloride, $0.01 \%$ sodium deoxycholate and $0.02 \% \mathrm{NP} 40$ ] at $34^{\circ} \mathrm{C}$ for $4 \mathrm{~h}$ or more. Sections were washed with $\mathrm{PBS} / 2 \mathrm{mmol} / \mathrm{l}$ EDTA and counterstained with eosin before mounting.

\section{Results}

Establishment of primary human mammary epithelia cells. The primary mammary epithelial cells HMEC 1 grew from a normal breast tissue biopsy after about 3 weeks in culture (Fig. 1A). The cells were contact inhibited, with a cobble stonelike morphology (Fig. 1B) and expressed cytokeratin (Fig. 1C), characteristics of epithelial cells. HMEC1 proliferated in culture for 6-10 passages and then underwent senescence and death (Fig. 1D). A second normal breast cell culture, HMEC2, was established and behaved similarly.

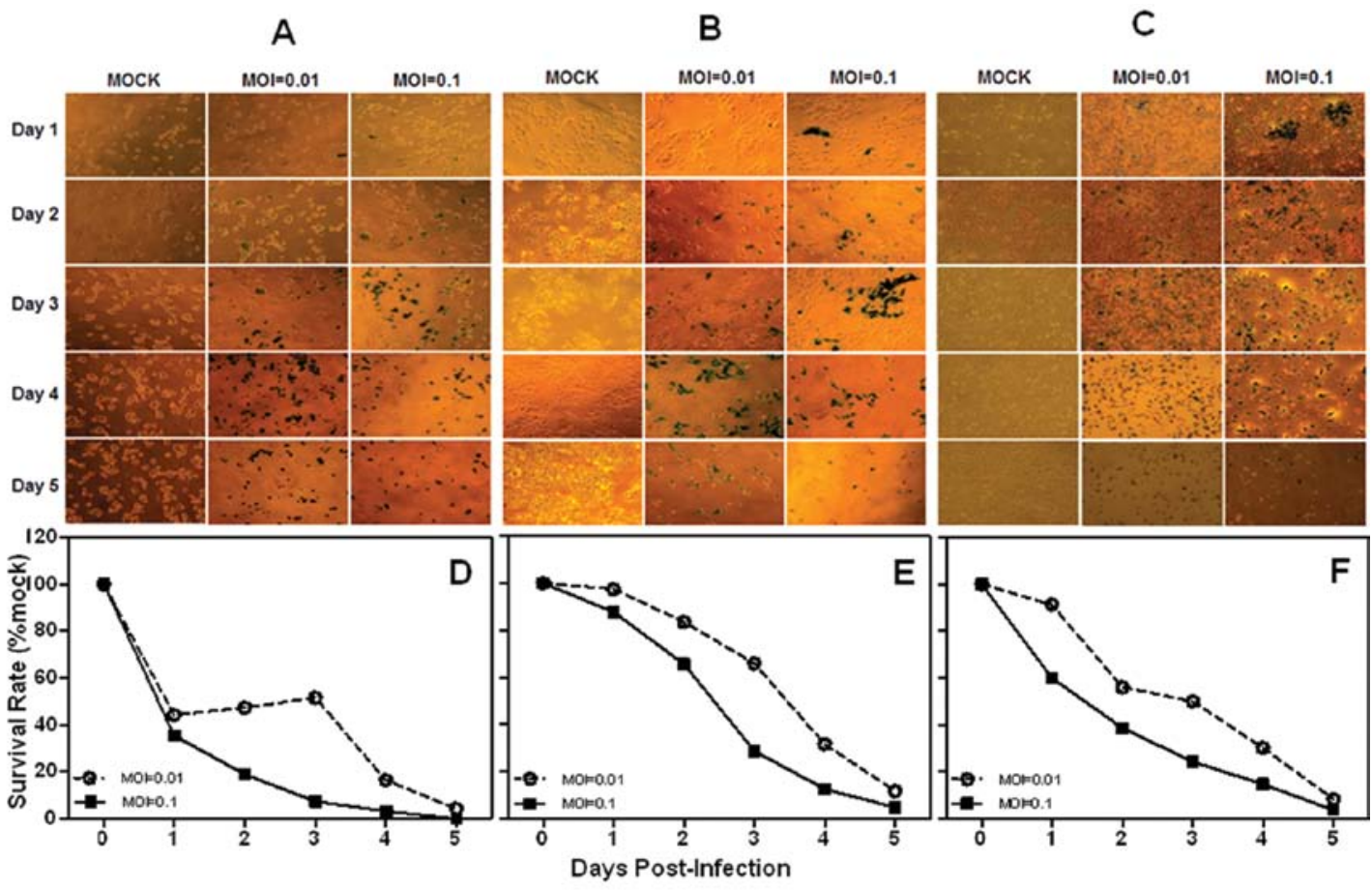

Figure 2. Susceptibility of human breast cancer cell lines to G47D in vitro. X-gal staining of MDA-MB-453 (A), MCF-7 (B) and SK-BR-3 (C) cells infected

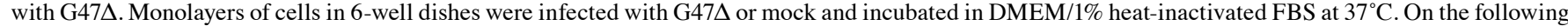
5 days after infection, the cells were fixed with cold $0.2 \%$ paraformaldehyde and stained with X-gal solution. Infected cells expressing LacZ stain positive with X-gal (blue). Monolayers of MDA-MB-453 (D), MCF-7 (E) and SK-BR-3 (F) cells in 6-well dishes were infected with G47A (MOI=0.01 or 0.1) or mock, incubated in DMEM/1\% heat-inactivated FBS at $37^{\circ} \mathrm{C}$ and counted on a hemocytometer on the days indicated. The average number of cells (from duplicate wells) is plotted as percent of the mock wells. 
A
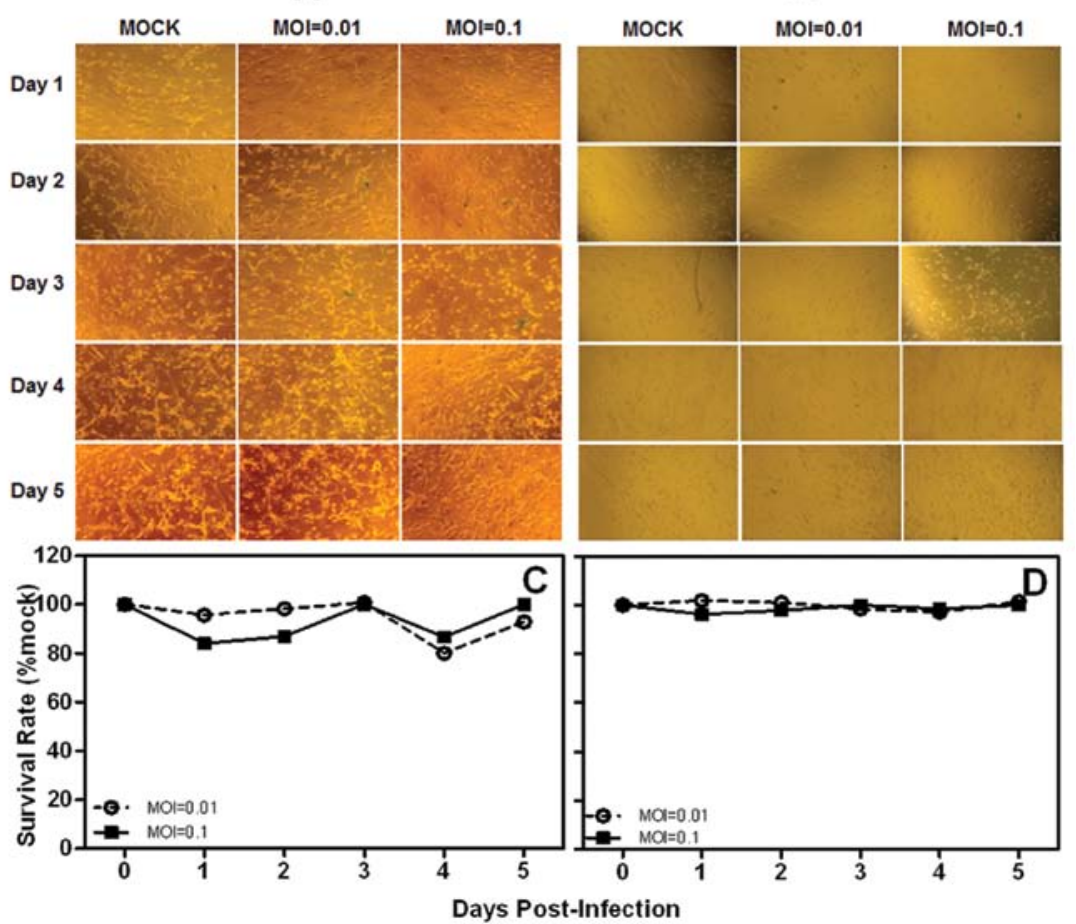

Figure 3. Susceptibility of cultured primary normal breast cells. X-gal staining of HMEC1 (A) and HMEC2 (B) cells infected with G47D. Monolayers of cells in 6-well dishes were infected with G47 0 or mock and incubated in keratinocyte/serum-free medium at $37^{\circ} \mathrm{C}$. The following 5 days after infection, the cells were fixed with cold $0.2 \%$ paraformaldehyde and stained with X-gal solution. Infected cells expressing LacZ stain positive with X-gal (blue). Monolayers of HMEC1 (C) and HMEC2 (D) cells in 6-well dishes were infected with G47D (MOI=0.01 or 0.1) or mock, incubated in keratinocyte/serum-free medium at $37^{\circ} \mathrm{C}$ and counted with a hemocytometer on the days indicated. The average number of cells (from duplicate wells) is plotted as percent of the mock wells.

A
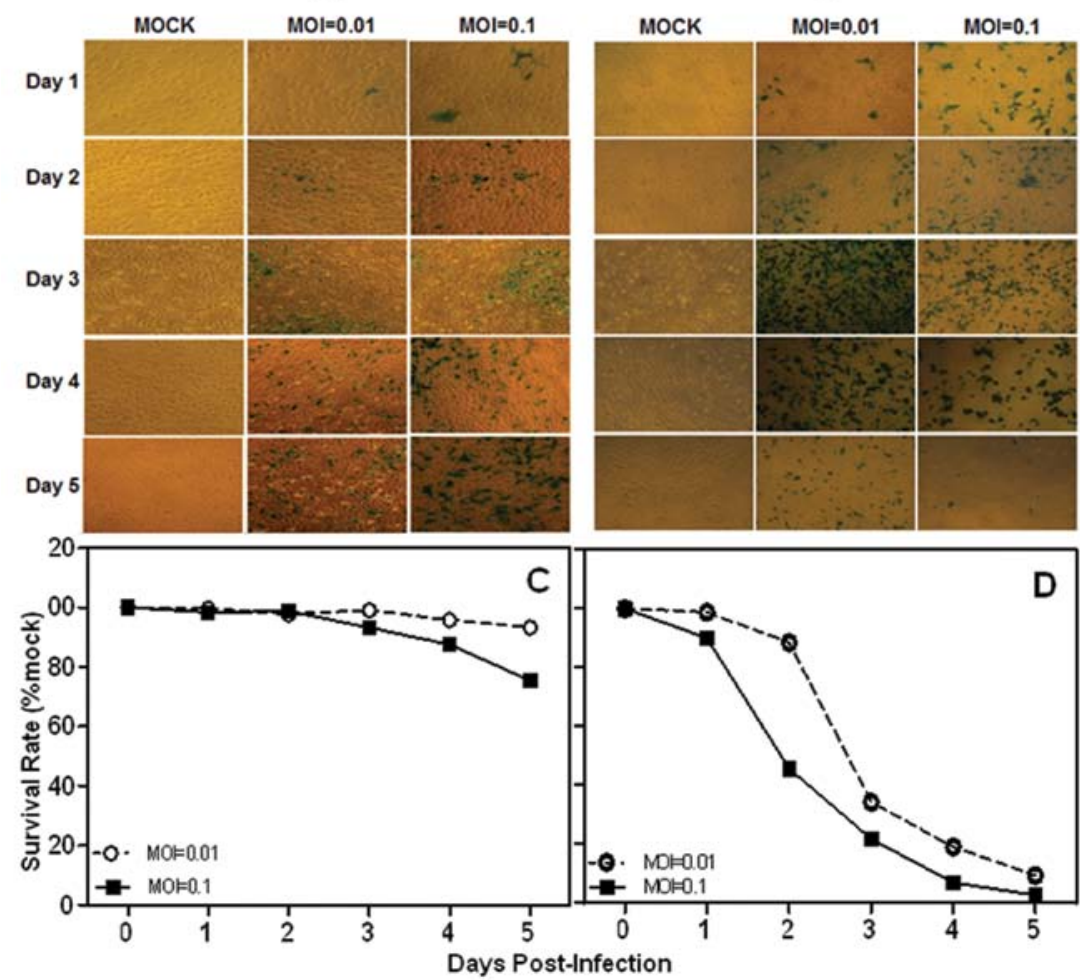

Figure 4. Susceptibility of immortalized breast cells. X-gal staining of MCF-10A (A) and 76N-tert (B) cells infected with G47D. Monolayers of cells in 6-well dishes were infected with $\mathrm{G} 47 \Delta$ or mock and incubated in keratinocyte/serum-free medium at $37^{\circ} \mathrm{C}$. The following 5 days after infection, the cells were fixed with cold $0.2 \%$ paraformaldehyde and stained with X-gal solution. Infected cells expressing LacZ stain positive with X-gal (blue). Monolayers of MCF-10A (C) and $76 \mathrm{~N}$-tert (D) cells in 6-well dishes were infected with $\mathrm{G} 47 \Delta$ (MOI=0.01 or 0.1 ) or mock, incubated in keratinocyte/serum-free medium at $37^{\circ} \mathrm{C}$ and counted by a hemocytometer on the days indicated. The average number of cells (from duplicate wells) is plotted as percent of the mock wells. 

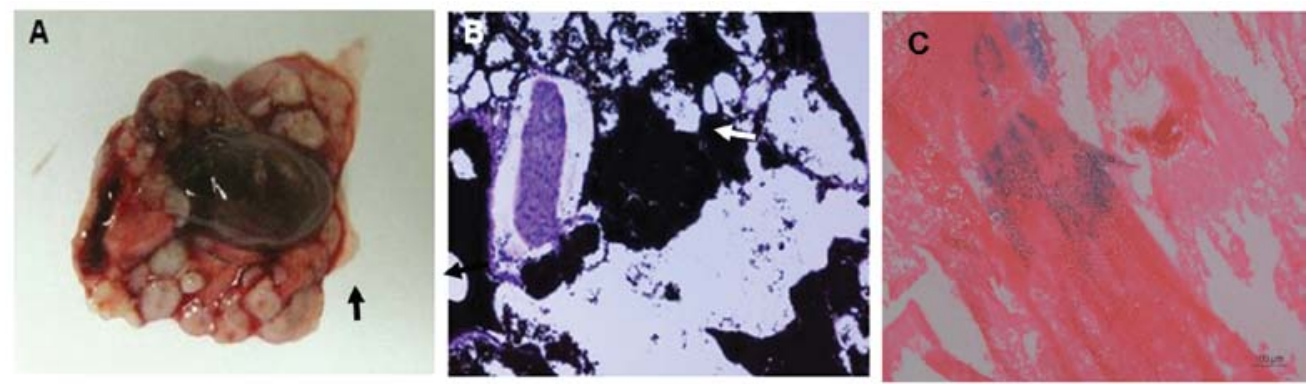

Figure 5. Established lung metastatic breast tumors infected with G47 the tail vein and the lungs were excised on day 56 (arrows point to tumor nodules). (B) H\&E staining of Indian ink stained lungs harboring an MDA-MB-435 metastatic tumor (arrow point to the metastasis breast tumor). (C) Coronal section through the lung after G47 $\left(2 \times 10^{7}\right.$ pfu) injection. Sections were stained with X-gal to identify cells containing replicating G47 $\Delta$ (blue) and counterstained with eosin.

In vitro cytotoxicity. To assess the specificity of oncolytic HSV G47 $\Delta$ for breast cancer cells, normal breast epithelia cells (HMEC1 and HMEC2), immortalized breast cells (MCF-10A and $76 \mathrm{~N}$-tert) and breast cancer cells (SK-BR-3, MDA-MB453 and MCF-7) were infected with $\mathrm{G} 47 \Delta$ at $\mathrm{MOI}=0.01$ and 0.1 . For all of the breast cancer cell lines, $>85$ and $95 \%$ of cells were killed at MOI $=0.01$ and 0.1 , respectively, by the fifth day of infection (Fig. 2D-F).

Conversely, normal breast cells (HMEC1 and HMEC2) were viable 5 days after infection (Fig. 3C, D). The immortalized breast cell line $76 \mathrm{~N}$-tert was similarly sensitive as the breast cancer cell lines (Fig. 4D), whereas only a slight decrease was observed with MCF-10A, about $25 \%$ of cells were killed at MOI=0.1 on Day 5 (Fig. 4C). G47 $\Delta$ infected cells express LacZ and thus can be stained by X-gal histochemistry. In vitro spread of G47 $\Delta$ correlated with cytotoxicity (blue cells; Figs. 2A-C and 4A, B), so that only isolated normal breast cells stained positive (Fig. 3A, B).

Efficacy of systemic G474 therapy on pulmonary metastatic breast cancer. In prior studies, we demonstrated that G47 $\Delta$ was cytotoxic in MDA-MB-435 cells, and that intratumoral injection of G47 $\Delta$ was effective in treating MDA-MB-435 breast tumor xenografts, with some subcutaneous tumors regressing completely (14).

To evaluate the therapeutic efficacy of G47 $\Delta$ in advanced breast cancer, we established a metastatic lung tumor model with human MDA-MB-435 breast cancer cells (Fig. 5A), which were treated by systemic administration of G47 $\Delta$ via tail vein injection. To evaluate $\mathrm{G} 47 \Delta$ replication in vivo, tumor-bearing mice were treated with G47 $\Delta$ on Days 44 and 47 post-tumor implantation, sacrificed on Days 48, 50, 53, 56 and 59, and $\mathrm{X}$-gal histochemistry performed on sectioned lungs to detect G47 $\Delta$-infected cells. Large numbers of X-gal-positive tumor cells were observed (Fig. 5C, blue).

To evaluate efficacy, mice with established lung metastases were injected i.v. with G47A or PBS on Days 14, 17, 21 and 24 and sacrificed on Day 56, with the lungs then excised, weighed and stained with India ink. Due to tumor burden, the mean lung weight of the mock-treated group was significantly heavier than that of the G47D-treated group (Fig. 6C). The average number of tumor nodules on the lung surface of G47D-treated mice significantly reduced by over 9 -fold (Fig. 6A, D). In addition, the mock-treated mice had extrapleural tumors attached to

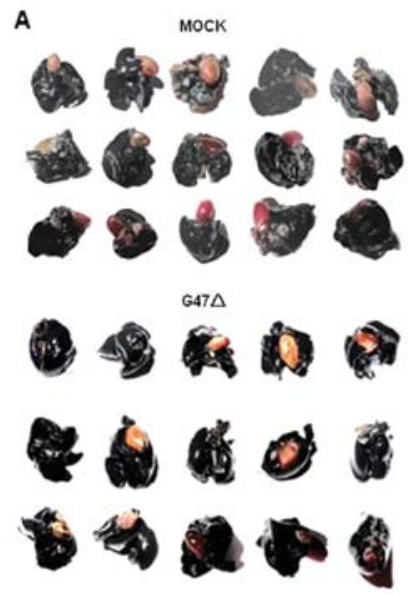

B

D

Mean Tumor Nodules
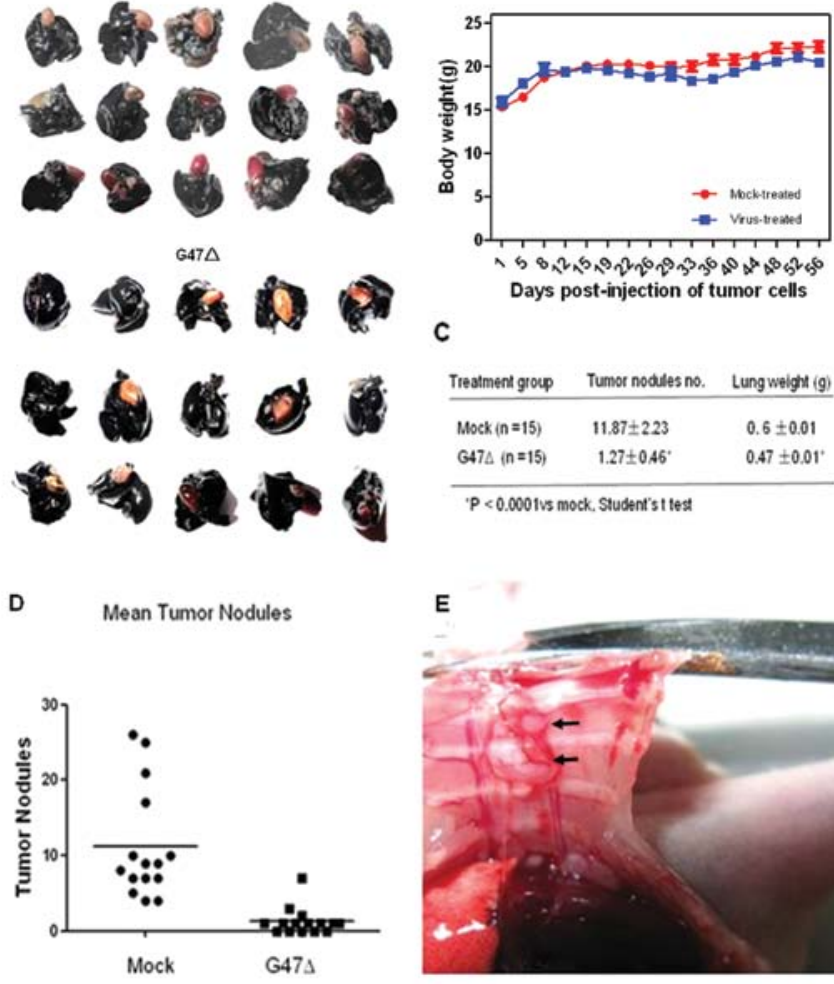

C

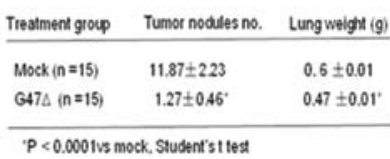

$p<0.000$ ivs mock. Student'st test

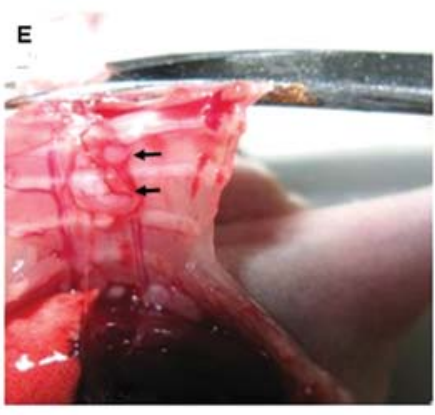

Figure 6. Efficacy of i.v. oncolytic HSV treatment of established MDAMB-435 lung tumors in Balb/c nude mice. Mice were treated with G47A ( $2 \times 10^{7} \mathrm{pfu} / 100 \mu \mathrm{l}$; i.v.) or virus buffer (mock), on Days 14, 17, 21 and 24 after implantation of MDA-MB-435. (A) Photographs of lungs from the G47A(lower) and mock-treatment (upper) groups stained with India ink: white masses on the surface of the lungs represent tumor nodules. (B) Body weight of mice in the G47 $\Delta$-treated group and mock-treated group was measured twice a week. There was no significant difference in mean body weight between the two groups ( $\mathrm{P}=0.192$, independent-sample $\mathrm{t}$ test). (C) The mean number of tumor nodules on the surface of lungs and mean lung weights of mice in the Mock or G47 $\Delta$-treatment groups. (D) Tumor nodule count for each mouse. G47 $\Delta$ significantly reduced the number of tumor nodules compared with mock $(\mathrm{P}<0.0001$, Student's t-test). (E) Mock-treated mice had extrapleural tumors that were attached to the rib cage (arrows point to the breast tumor metastases).

the rib cage (Fig. 6E). The body weight of the mice in the two groups did not significantly differ; suggesting a lack of virus induced adverse effects (Fig. 6B). 


\section{Discussion}

The criteria for engineering replication-competent oncolytic HSV-1 vectors are to target tumor cells for death while sparing normal tissue from damage, thus providing a safe and efficacious approach for cancer therapy. Oncolytic HSV-1 vectors have been developed with mutations in genes affecting nucleotide and protein metabolism, neuropathogenicity, and immuneevasiveness, to generate tumor selectivity, efficacy and safety (17). Tumors are typically heterogeneous and develop resistance to chemotherapeutic agents, such that no single therapy is likely to be universally applicable or completely effective for cancer treatment. Oncolytic HSV-1 vectors act differently from routine therapeutic agents such as chemotherapy and radiotherapy and thus provide an additional strategy for treatment.

In this study, we investigated $\mathrm{G} 47 \Delta$, a third generation oncolytic HSV-1 vector derived from G207, with an additional deletion of $\alpha 47$ and the promoter region of US11, which enhances its growth and the immune recognition of infected cells (12). These multiple mutations make the occurrence of a pathogenic revertant extremely inprobable. The cytotoxic and oncolytic effects of G47 $\Delta$ should be confined to tumor cells, however, it is important to demonstrate that G47 $\Delta$ cannot replicate in normal cells. We show that human normal primary breast epithelial cells remain viable 5 days after infection by G47D, whereas more than $95 \%$ of the cells of three human breast cancer cell lines, SK-BR-3, MDA-MB-453 and MCF-7, were killed in this time.

Clinical trials, with no serious adverse events attributable to the virus, have been completed with G207 and 1716 in patients with recurrent malignant glioma who were refractory to conventional treatments of radiation and chemotherapy, as well as NV1020 in patients with liver metastases of colorectal cancer, HF10 in patients with breast cancer and OncoVex ${ }^{\mathrm{GMCSF}}$ in patients with accessible metastases $(18,19)$. Recently, G47 $\Delta$ has entered phase I clinical trial for progressive glioblastoma (Ino Y, 2009). WHO International Clinical Trials Registry http://apps.who.int/ trialsearch/trial.aspx?trialid=JPRN-UMIN000002661). Our preclinical studies on G47 $\Delta$ provide support for its use in clinical trials for breast cancer treatment.

Bypass of senescence and immortalization are considered early steps in tumor development. We show that G47 $\Delta$ was highly cytotoxic to hTERT-transduced immortalized breast $76 \mathrm{~N}$-tert cells in vitro at low MOIs, with more than $85 \%$ of cells killed at a MOI of 0.01 , whereas only a slight effect was observed with spontaneously immortalized breast MCF-10A cells (7\% of cells). This may indicate that $76 \mathrm{~N}$-tert cells are further along a transformation pathway than MCF-10A cells and that the early stage phenotype of the $76 \mathrm{~N}$-tert cells is responsive to G47D replication. Cancer metastasis to distant sites is the primary factor contributing to cancer-related deaths. From 21 to $33 \%$ of all lymph nodenegative breast cancer patients subsequently develop distant metastases (20-26). Even patients with small primary tumors have a 15-25\% chance of developing distant metastases (27). Metastatic lesions are incurable in most patients, with the predominant metastatic proclivity of breast cancer being to the lung, bone, liver and brain (27).

In this study, with an established pulmonary metastatic breast cancer model, oncolytic HSV-1 G47 $\Delta$ was administered twice weekly for two weeks without observable toxicity in the animals, while significantly inhibiting MDA-MB-435 lung tumor nodules as compared with mock therapy. This study is the first to evaluate the efficacy of systemic G47 $\Delta$ for the treatment of lung metastases of human breast cancer.

In conclusion, oncolytic HSV G47 $\Delta$ was effective against human breast cancer and select immortalized breast cells without killing normal breast cells. The systemic administration of G47 $\Delta$ via tail vein injection successfully controlled pulmonary metastatic breast cancer. Further investigation and consideration of clinical application are warranted for this novel therapy for breast cancer.

\section{Acknowledgements}

This study was supported by the NSF of China (30672410) and the Guangdong Natural Science Foundation (06104599).

\section{References}

1. Mullen JT and Tanabe KK: Viral oncolysis. Oncologist 7: 106-119, 2002.

2. Martuza RL, Malick A, Markert JM, Ruffner KL and Coen DM: Experimental therapy of human glioma by means of a genetically engineered virus mutant. Science 252: 854-856, 1991.

3. Chou J, Kern ER, Whitley RJ and Roizman B: Mapping of herpes simplex virus-1 neurovirulence to gamma 134.5 , a gene nonessential for growth in culture. Science 250: 1262-1266, 1990.

4. Chou J and Roizman B: The gamma 1(34.5) gene of herpes simplex virus 1 precludes neuroblastoma cells from triggering total shutoff of protein synthesis characteristic of programed cell death in neuronal cells. Proc Natl Acad Sci USA 89: 3266-3270, 1992.

5. Carroll NM, Chiocca EA, Takahashi K and Tanabe KK: Enhancement of gene therapy specificity for diffuse colon carcinoma liver metastases with recombinant herpes simplex virus. Ann Surg 224: 323-330, 1996.

6. Mineta T, Rabkin SD and Martuza RL: Treatment of malignant gliomas using ganciclovir-hypersensitive, ribonucleotide reductase-deficient herpes simplex viral mutant. Cancer Res 54: 3963-3966, 1994.

7. Toda M, Rabkin SD, Kojima H and Martuza RL: Herpes simplex virus as an in situ cancer vaccine for the induction of specific anti-tumor immunity. Hum Gene Ther 10: 385-393, 1999.

8. Thomas DL and Fraser NW: HSV-1 therapy of primary tumors reduces the number of metastases in an immune-competent model of metastatic breast cancer. Mol Ther 8: 543-551, 2003.

9. Liu BL, Robinson M, Han ZQ, et al: ICP34.5 deleted herpes simplex virus with enhanced oncolytic, immune stimulating, and anti-tumour properties. Gene Ther 10: 292-303, 2003.

10. York IA, Roop C, Andrews DW, Riddell SR, Graham FL and Johnson DC: A cytosolic herpes simplex virus protein inhibits antigen presentation to CD8 ${ }^{+} \mathrm{T}$ lymphocytes. Cell 77: 525-535, 1994.

11. Huard B and Fruh K: A role for MHC class I down-regulation in NK cell lysis of herpes virus-infected cells. Eur J Immunol 30: 509-515, 2000

12. Todo T, Martuza RL, Rabkin SD and Johnson PA: Oncolytic herpes simplex virus vector with enhanced MHC class I presentation and tumor cell killing. Proc Natl Acad Sci USA 98: 6396-6401, 2001.

13. Mohr I and Gluzman Y: A herpesvirus genetic element which affects translation in the absence of the viral GADD34 function. EMBO J 15: 4759-4766, 1996.

14. Liu RB and Rabkin SD: Oncolytic herpes simplex virus vectors for the treatment of human breast cancer. Chin Med J (Engl) 118: 307-312, 2005.

15. Liu R, Varghese S and Rabkin SD: Oncolytic herpes simplex virus vector therapy of breast cancer in C3(1)/SV40 T-antigen transgenic mice. Cancer Res 65: 1532-1540, 2005.

16. Song LB, Zeng MS, Liao WT, et al: Bmi-1 is a novel molecular marker of nasopharyngeal carcinoma progression and immortalizes primary human nasopharyngeal epithelial cells. Cancer Res 66: 6225-6232, 2006. 
17. Varghese S and Rabkin SD: Oncolytic herpes simplex virus vectors for cancer virotherapy. Cancer Gene Ther 9: 967-978, 2002.

18. Aghi M and Martuza RL: Oncolytic viral therapies - the clinical experience. Oncogene 24: 7802-7816, 2005

19. Kanai R, Wakimoto H, Cheema T and Rabkin SD: Oncolytic herpes simplex virus vectors and chemotherapy: are combinatorial strategies more effective for cancer? Future Oncol 6 : 619-634, 2010.

20. Heimann R, Lan F, McBride R and Hellman S: Separating favorable from unfavorable prognostic markers in breast cancer: the role of E-cadherin. Cancer Res 60: 298-304, 2000.

21. Quiet CA, Ferguson DJ, Weichselbaum RR and Hellman S: Natural history of node-negative breast cancer: a study of 826 patients with long-term follow-up. J Clin Oncol 13: 1144-1151, 1995.

22. Rosen PR, Groshen S, Saigo PE, Kinne DW and Hellman S: A long-term follow-up study of survival in stage I (T1NOM0) and stage II (T1N1M0) breast carcinoma. J Clin Oncol 7: 355-366, 1989.
23. Tan LK, Giri D, Hummer AJ, et al: Occult axillary node metastases in breast cancer are prognostically significant: results in 368 node-negative patients with 20 -year follow-up. J Clin Oncol 26: 1803-1809, 2008.

24. Wang Y, Klijn JG, Zhang Y, et al: Gene-expression profiles to predict distant metastasis of lymph-node-negative primary breast cancer. Lancet 365: 671-679, 2005.

25. Clare SE, Sener SF, Wilkens W, Goldschmidt R, Merkel D and Winchester DJ: Prognostic significance of occult lymph node metastases in node-negative breast cancer. Ann Surg Oncol 4: 447-451, 1997.

26. Heimann R and Hellman S: Clinical progression of breast cancer malignant behavior: what to expect and when to expect it. J Clin Oncol 18: 591-599, 2000.

27. Pagani O, Senkus E, Wood W, et al: International guidelines for management of metastatic breast cancer: can metastatic breast cancer be cured? J Natl Cancer Inst 102: 456-463, 2010. 